\title{
Post-Traumatic Stress Outcomes in Non-Hodgkin's Lymphoma Survivors
}

\author{
Sophia K. Smith, Sheryl Zimmerman, Christianna S. Williams, John S. Preisser, and \\ Elizabeth C. Clipp \\ University of North Carolina at Chapel Hill, Chapel Hill; Duke University, Durham, NC.
}

\begin{abstract}
Purpose-A large body of evidence suggests that being diagnosed with and treated for cancer adversely affects functioning and quality of life, yet less is known about longer term outcomes. Therefore, this study aims to estimate the prevalence of post-traumatic stress disorder (PTSD) symptoms in survivors of adult non-Hodgkin's lymphoma (NHL) who are at least 2 years postdiagnosis and identify the risk factors associated with PTSD symptoms, with a focus on those that are amenable for screening and modifiable.
\end{abstract}

Patients and Methods-A total of 886 NHL survivors identified from the cancer registries of two hospitals in North Carolina participated, ranging in age from 25 to 92 years old and ranging from 2 to 44 years postdiagnosis. Survivors were mailed a survey that assessed PTSD symptoms and quality of life.

Results-Participants averaged 10.2 years postdiagnosis, and most (61\%) reported no PTSD symptoms. The adjusted prevalence for full PTSD was $7.9 \%$, with an additional $9.1 \%$ meeting criteria for partial PTSD. Modifiable risk factors that were independently associated with PTSD in multiple linear regression included less social support, negative appraisals of life threat and treatment intensity, and more employment and insurance issues. Additionally, several demographic characteristics (nonwhite race, less education, and younger age) and clinical or health-related factors (active disease, more recent diagnosis, and more comorbidity) were independently associated with PTSD.

Conclusion-Although only $8 \%$ of survivors met PTSD diagnostic criteria, the impact of a cancer diagnosis and treatment persists for many survivors, as evidenced in 39\% of this sample. Early identification of those at risk could enable treatment to minimize PTSD symptomatology.

Copyright $\odot 2008$ American Society of Clinical Oncology. All rights reserved.

Corresponding author: Sophia Smith, PhD, Cecil Sheps Center for Health Services Research, Campus Box 7590, 725 Martin Luther King Jr Blvd, Chapel Hill, NC 27599-7590; sophia_smith@unc.edu..

AUTHOR CONTRIBUTIONS Conception and design: Sophia K. Smith, Sheryl Zimmerman, Christianna S. Williams, John S.

Preisser, Elizabeth C. Clipp

Administrative support: Sophia K. Smith

Collection and assembly of data: Sophia K. Smith

Data analysis and interpretation: Sophia K. Smith, Sheryl Zimmerman, Christianna S. Williams, John S. Preisser, Elizabeth C. Clipp

Manuscript writing: Sophia K. Smith, Sheryl Zimmerman, Christianna S. Williams, John S. Preisser, Elizabeth C. Clipp

Final approval of manuscript: Sophia K. Smith, Sheryl Zimmerman, Christianna S. Williams, John S. Preisser, Elizabeth C. Clipp

Presented in part at the 11th Annual Society for Social Work Research Conference, January 11-14, 2007, San Francisco, CA, and at the National Cancer Institute/American Cancer Society Cancer Survivorship Research Conference, October 4-6, 2006, Bethesda, MD. AUTHORS' DISCLOSURES OF POTENTIAL CONFLICTS OF INTEREST The author(s) indicated no potential conflicts of interest. 


\section{INTRODUCTION}

A growing body of evidence suggests that the experience of being diagnosed with and treated for cancer, regardless of age, adversely affects functioning and quality of life. However, less is known about the longer term effects ( 2 years after diagnosis and beyond) of cancer on survivors' well-being, especially adults with cancer other than breast cancer. Of note, little is known about the needs of individuals diagnosed with non-Hodgkin's lymphoma (NHL), which is the sixth most common cancer in the United States and has an overall 5-year survival rate of $63 \%$.

NHL incidence rates have doubled since the early 1970 s, an increase that is only partially explained by AIDS-related NHL. The lifetime risk for developing NHL is approximately one in 50, with older adults (median age of 66 years) at highest risk. ${ }^{1}$ Consequently, and because the number of survivors in the United States is rapidly increasing as a result of both medical advances that treat other conditions and the aging of the population, health care teams would benefit from evidence regarding the needs of NHL survivors. If indicated, interventions could be provided to improve their well-being.

Post-traumatic stress disorder (PTSD) is a set of symptoms (re-experiencing, avoidance, and arousal) following direct exposure to a traumatic stressor where the individual is involved in, witnesses, or learns about threatened death or serious injury. Symptoms of PTSD, such as re-experiencing distressing events, avoiding cancer-related experiences, and physiologic arousal, have been reported by breast cancer survivors, with prevalence estimates ranging from 5\% to 6\%. ${ }^{2-} 5$ Unfortunately, little data are available regarding PTSD in other adult cancers. A conceptual modelfor cancer-related PTSD is illustrated in Figure 1, in which the cancer diagnosis and associated events such as treatment and general health status are conceptualized as stressors. Demographic characteristics and psychosocial resources relate to the stressors, and these three sets of variables can directly or indirectly influence PTSD. This model is based on the stress, appraisal, and coping theory of Lazarus and Folkman, ${ }^{6}$ which emphasizes the relationship between the person (his/her characteristics) and his/her environment (cancer experience). Of note, cancer-related PTSD differs from more traditional traumas in that the intrusions tend to be future-oriented fears, such as concerning recurrence or treatment. The model reflects risk factors identified in previous studies of adult cancer survivors, including younger age, ${ }^{7}$ female gender and negative perceptions of treatment intensity, 8 lower income, 2 less social support and education, 3 and less time since treatment and advanced disease. ${ }^{4}$ This article presents prevalence estimates and risk factors for PTSD, with a particular focus on potentially modifiable factors.

\section{PATIENTS AND METHODS}

\section{Participants and Procedures}

Potential study participants were identified through tumor registries at the Duke and University of North Carolina at Chapel Hill Lineberger Comprehensive Cancer Centers. Patients were eligible if they were diagnosed with NHL, were at least 19 years old at diagnosis, and were at least 2 years postdiagnosis, whether or not they had currently active disease. Each patient's physician granted approval before the researchers requested patient participation. Approval for all procedures was granted by the Institutional Review Boards at the University of North Carolina and Duke University Schools of Medicine. On the basis of the method for mailed surveys of Dillman, ${ }^{9}$ prospective participants were sent a brief prenotice letter, a self-administered questionnaire with a $\$ 2$ bill incentive and other studyrelated materials, and thank you/reminder postcards. Nonrespondents were sent replacements and later telephoned to confirm receipt of the mailed survey packet. 


\section{Measures}

Demographic and clinical characteristics-Self-reported participant demographic information included birth date, sex, race, ethnicity, marital status, income, and education. Details regarding diagnosis and treatment were obtained from Tumor Registry databases. Histology was categorized as indolent or aggressive based on the updated Revised European American Lymphoma/WHO classification system. ${ }^{10}$

Health status-The Self-Administered Comorbidity Questionnaire, a 12-item self-report version of the Charlson Index, was used to assess other health-related problems. ${ }^{11}$ An individual can receive up to 3 points for each of 12 medical conditions (1 point each for presence of the problem, current treatment, and functional limitation). Selected questions related to health care use and secondary cancer status were adapted from the Childhood Cancer Survivor Study survey, a large epidemiologic study of long-term survivors of childhood cancer. ${ }^{12}$

Psychosocial-Perceived availability of social support was assessed using the 20-item Medical Outcomes Study-Social Support Survey. ${ }^{13}$ It has been used in various populations, including long-term breast cancer survivors. ${ }^{3,14}$ The standardized score ranges from 20 to 100 and yielded an $\alpha=.97$ in this study. The Appraisal of Life Threat and Treatment Intensity Questionnaire assesses the extent to which cancer and its treatment are perceived to be life threatening and intense in the past and currently. ${ }^{15}$ Its six items sum to a score ranging from 6 to 30 and yielded an $\alpha=.80$ in the present study. Finally, to assess employment and insurance-related situations and difficulties, 24 items (possible score range, 0 to $24 ; \alpha=.82$ ) were derived from an instrument developed by the Cancer and Leukemia Group B clinical research group. ${ }^{16}$ Examples include "I did not change jobs for fear of losing health insurance" and "I was encouraged to leave my job" because of the cancer.

Post-traumatic stress-The PTSD Checklist-Civilian Version (PCL-C) assesses symptomatology in noncombat populations by presenting a self-report symptom checklist that closely mirrors criteria set forth by the Diagnostic and Statistical Manual of Mental Disorders, Fourth Edition for a formal diagnosis of PTSD.17,18 The instructions were modified so that symptoms were keyed to the particular traumatic stressor of interest; specifically, survivors were asked to rate each PTSD symptom in the past 4 weeks with respect to their diagnosis and treatment for lymphoma. Each of 17 symptoms is rated with respect to intensity on a scale of 1 (not at all) to 5 (extremely bothersome), and item scores are summed to create a total that can range from 17 to 85 points. The following two approaches were used to identify PTSD: (1) the cutoff method, in which those with scores $\geq$ 44 are classified as having PTSD19; and (2) the symptom cluster method, which follows the Diagnostic and Statistical Manual of Mental Disorders, Fourth Edition criteria for PTSD in that individuals who report having been at least moderately bothered by (score $\geq 3$ ) one or more re-experiencing symptoms (of five symptoms; eg, nightmares), three or more avoidance symptoms (of seven symptoms; eg, evading follow-ups), and two or more arousal symptoms (of five symptoms; eg, easily startled) are classified as having PTSD.17 Using the PCL-C cutoff of 44, Blanchard et al19 reported a sensitivity of 0.94 and specificity of 0.86 with the Clinician Administered PTSD Scale. In other work, the PCL-C demonstrated an $\alpha$ $=.97$, test-retest reliability of 0.96 , and convergent validity of 0.93 with the Mississippi Scale in a sample of 123 Vietnam veterans. ${ }^{18}$ In this study, the total score yielded an $\alpha=.91$, and the subscales produced the following internal consistency: re-experiencing, $\alpha=.88$; avoidance, $\alpha=.82$; and arousal, $\alpha=.78$. 


\section{Statistical Methods}

To assure that the PTSD prevalence estimates reflected the Tumor Registry sampling frame, sample prevalence estimates were adjusted for nonresponse based on race (African American or non-African American), as well as current age and age at diagnosis (in 20-year increments). Although the data set overall had a small amount of missing data, the level of missingness in three variables, namely income (10.6\%), stage (13.5\%), and disease status (10.9\%), justified multiple imputation via the Markov chain Monte Carlo algorithm. ${ }^{20}$ All variables in the conceptual model were included in the Markov chain Monte Carlo algorithm, but imputed values for the outcome variable (PCL-C) were not generated. Twenty data sets containing imputed values were used in the multiple linear and logistic regression analyses, and SEs adjusted for imputation were estimated in the SAS MIANALYZE procedure (SAS Institute, Cary, NC).21 Bivariate analyses compared mean levels of PCL-C for those with and without a given potential risk factor using $t$ tests and analysis of variance. Pearson correlations were calculated between the PCL-C and all continuous independent variables. Multiple linear regression was used to estimate the independent associations between candidate variables and the continuous PCL-C score; logistic regression was used to estimate the associations of the same variables with meeting criteria for full or partial PTSD, as defined by the symptom cluster method. Variables were selected for inclusion in the multivariable models if they were at least marginally significant $(P<.10)$ in bivariate analyses. Data management and bivariate analyses were carried out with SPSS Version 14.0 (SPSS Inc, Chicago, IL). Multiple imputation and multivariable analyses were conducted using SAS Version 9.1.3 (SAS Institute).

\section{RESULTS}

Of the 1,312 eligible survivors who were mailed a survey, 117 (9\%) of the packages were returned undelivered, and tracing attempts were unsuccessful. Of the remaining 1,195 survivors who were assumed to have received a survey, 886 (74\%) completed and returned their surveys, $258(22 \%)$ did not respond, and 51 (4\%) refused participation. Sample bias analyses using demographic information from the registries indicated that participating survivors, compared with nonparticipants, were less frequently African American (10\% $\mathrm{v}$ $20 \%$, respectively; $P<.001$ ), older at study enrollment (mean age, $62.9 v 58.8$ years, respectively; $P<.001$ ), and older at diagnosis (52.6 $v 48.1$ year, respectively; $P<.001)$. The 868 survivors who completed the PCL-C were included in further analyses.

The sample characteristics are listed in Table 1. A similar number of females and males participated, $14 \%$ were nonwhite, $25 \%$ earned less than $\$ 30,000$ annually, 38\% had a college degree, and $40 \%$ were employed. Mean age at study enrollment was 63 years, and almost half of participants (46\%) were older adults ( $\geq 65$ years of age). The majority of participants reported having received chemotherapy treatment (78\%) and that they were not currently in treatment for NHL (85\%). The mean age at lymphoma diagnosis was 53 years (range, 19 to 87 years). The mean interval from diagnosis to study enrollment was 10.2 years (range, 2 to 44 years). Having received a second primary cancer diagnosis was cited by $14 \%$ of the sample, and $4 \%$ were currently receiving treatment for it. Participants cited an average of 2.9 comorbid conditions (standard deviation [SD], 2.1 comorbidities; range, 0 to 12 comorbidities), primarily back pain (42\%), high blood pressure (40\%), and heart disease (26\%), and $1 \%$ had AIDS-related NHL. Scores on the psychosocial variables were as follows: for social support, 83.1 (SD, 16.4; range, 20 to 100); appraisal of life threat, 19.3 (SD, 6.0; range, 6 to 30); and cancer-related employment and insurance issues, 1.1 (SD, 2.1; range, 0 to 17).

The overall mean PCL-C score in this sample was 27.0 (SD, 9.9), with subscale scores of $6.9(\mathrm{SD}, 3.1)$ for re-experiencing, $9.3(\mathrm{SD}, 3.8)$ for arousal, and $10.8(\mathrm{SD}, 4.4)$ for 
avoidance. As shown in Table 2, the prevalence of cancer-related PTSD in the sample was similar using the cutoff score $(6.9 \%)$ and symptom cluster $(7.6 \%)$ methods. After applying weights to account for nonresponse bias, the adjusted prevalence rose to $7.4 \%$ and $7.9 \%$ for each of these methods. Seventeen percent rated at least two of the three PTSD symptom clusters (partial or full PTSD) as occurring moderately to extremely often. Across the symptom clusters, $30 \%$ met the criteria for arousal, $20 \%$ met criteria for re-experiencing, and $14 \%$ met criteria for avoidance. Overall, $39 \%$ met criteria for at least one PTSD symptom cluster.

\section{Relationship of PTSD Symptoms to Other Variables}

Bivariate associations between PCL-C scores and the independent variables are listed in Table 3. Among demographic and clinical variables, those who were nonwhite, had an annual income under $\$ 30,000$, did not obtain a college degree, were younger, received a bone marrow or stem-cell transplantation, received biologic treatment, were currently receiving treatment for NHL, had active disease, and experienced at least one NHL recurrence had higher PCL-C scores (all $P \leq .01$ ). Among the continuous variables, the strongest PCL-C relationships (all $P<.001)$ were for comorbidity $(r=0.27)$, social support $(r$ $=-0.36)$, appraisal of life threat and treatment intensity $(r=0.37)$, and cancer-related insurance and employment issues $(r=0.27)$. In addition, significant PCL-C associations were found for age at study enrollment $(r=-0.15)$, initial stage of disease $(r=0.11)$, sum of treatment types $(r=0.13)$, number of NHL-related visits to a physician $(r=0.19)$, age at diagnosis $(r=-0.08)$, time since diagnosis $(r=-0.11)$, and time since last physical examination $(r=0.08)$.

\section{Regression Analyses}

Table 4 lists the results of a multiple linear regression for the PCL-C score and logistic regression for partial or full PTSD (ie, at least two symptom clusters) for those variables at least marginally significant in the bivariate analyses $(P<.10)$. Nonwhite race, less education, younger age at enrollment, having active NHL disease, less time since diagnosis, more comorbidity, less social support, more negative appraisals, and more insurance and employment issues were independently associated with worse PCL-C scores. For example, survivors with active disease had PCL-C scores 3.7 points higher than those in remission or cured $(P=.001)$. Furthermore, survivors of nonwhite race had PCL-C scores 2.4 points higher than those of white race $(P=.004)$. The full model accounted for $38.5 \%$ of the variance $(P<.001)$.

In the logistic regression model, three risk factors (nonwhite race, younger age, and active disease status) that were identified in the linear regression were not independently associated with PTSD symptoms, although race remained marginally statistically significant $(P=.07)$, with nonwhite participants having 1.7 times greater odds of having partial or full PTSD than white participants. Furthermore, when a logistic model for full PTSD (symptom clusters = 3; $7.9 \%$ of sample) was estimated, the odds ratio was 2.8 (95\% CI, 1.4 to 5.8) for nonwhite race and 4.3 (95\% CI, 1.5 to 12.2) for active disease. Finally, education, time since diagnosis, comorbidity, and all psychosocial variables (social support, appraisals, and employment and insurance issues) had independent associations with PTSD symptoms.

\section{DISCUSSION}

This study is one of the first examinations of well-being among long-term survivors of NHL, who report varying degrees of PTSD symptomatology. Although the prevalence in this sample is somewhat lower than that found in some other traumatized populations, our adjusted prevalence of $7.4 \%$ to $7.9 \%$ is more than three times the $2.4 \%$ prevalence in the 
general adult population.22,23 Although prevalence estimates are highly dependent on the methodology and sample, our finding is similar to breast cancer survivors (for whom PTSD prevalence of 5\% to $6 \%$ has been reported). 3.4 Our prevalence was adjusted upwards by approximately $0.5 \%$ (from $6.9 \%$ to $7.4 \%$ ) when accounting for nonresponse, suggesting that similar and previous studies of cancer survivors may be under-reporting PTSD if they had response patterns similar to those in our study.

Although the majority of survivors (61\%) were not symptomatic for PTSD (ie, had no symptom clusters), the prevalence in certain subgroups is cause for concern. For example, nearly $16 \%$ of nonwhites qualified for a full PTSD diagnosis. The greater PTSD symptomatology among nonwhite participants persists after adjustment for other demographic and clinical factors, indicating that the discrepancy is not solely a result of differences in education or disease status. A possible explanation for this disparity is that individuals exposed to discrimination and prejudice may already be living with a heightened sense of arousal and greater stress.

In two other subgroups, $15 \%$ of those with active NHL disease and $12 \%$ of younger survivors ( $<50$ years of age) met criteria for PTSD. Survivors with current disease may experience daily reminders of their illness, such as pain, itchiness, and fever. In support of this finding, Deimling et $\mathrm{al}^{24}$ found that current cancer-related symptoms were the strongest predictor of hyperarousal in a study of 180 adult, long-term cancer survivors. However, it is worth noting that results from a multiple regression that excluded individuals with active disease in our sample were substantively the same. Regarding age, Kornblith et al7 suggest that younger survivors have less experience than their older counterparts in dealing with medical crises and life threats, thereby feeling more distressed overall. Furthermore, they may already be faced with more challenges at this earlier stage in the life cycle; for example, younger survivors had significantly more cancer-related employment and insurance issues than did their older counterparts (mean, $1.7 v 0.9$ issues, respectively; $P<.001$ ), which was largely attributable to the higher employment rate (75.8\% v 31.7\%, respectively). In addition to increasing age being a protective factor for PTSD, the passage of time seems to mitigate the impact of a cancer diagnosis and treatment. Perhaps least surprising was the finding that other health-related stressors (ie, comorbidities) were predictive of PTSD symptomatology.

It is encouraging to consider that the psychosocial factors related to PTSD are potentially modifiable. For example, cognitive behavioral and prolonged exposure techniques have been shown to be effective in reducing or eliminating PTSD symptoms in other traumatized populations, such as survivors of sexual assault, 25 but the efficacy of psychosocial treatments on cancer-related PTSD is just beginning to be examined. ${ }^{26}$ Interventions that combine therapeutic approaches (eg, cognitive behavioral therapy, support groups) may be desirable because we found social support to be correlated most strongly with avoidance symptoms $(r=-0.41, P<.001)$ and negative appraisals most related to re-experiencing symptoms $(r=0.36, P<.001)$.

This study has some limitations, including the absence of a comparison group, a crosssectional study design, and inclusion only of patients treated at two large comprehensive cancer centers. Without a comparison group, it is difficult to determine whether these NHL survivors had more or less PTSD symptomatology than a similar group of people who never had NHL. However, the markedly higher PTSD prevalence in our sample compared with general population estimates suggests that NHL survivors have elevated PTSD symptomatology, supporting the need to address it.

The cross-sectional design prevents determination of whether PTSD symptomatology or certain risk factors (eg, low social support) occurred first. However, it is reasonable to 
assume that many of the risk factors (eg, nonwhite race, less education) preceded the PTSD. Furthermore, this limitation should not affect the prevalence estimates except to the extent that the sample is a mix of individuals at different places in their course of survivorship. Finally, the inclusion of patients from only two large comprehensive cancer centers may limit the generalizability of our results to survivors living in other regions and treated at smaller hospitals. However, our demographic profile closely mirrors that of the national population of NHL survivors, thereby strengthening the robustness and generalizability of our prevalence estimates. Study strengths include a high response rate (74\%), adjustment for nonresponse, sophisticated and appropriate methodology for accounting for missing data, two methods (linear and logistic regression) for identifying PTSD risk factors, and a balanced sex ratio.

In closing, although the majority of survivors did not exhibit symptoms of PTSD, the impact of diagnosis and treatment for cancer persists for many, such as having physical reactions to reminders (trouble breathing or sweating), difficulties in concentration and sleep, detachment from others, and avoiding medical care. Identification of those at risk (eg, nonwhite, less education) early in the survivorship trajectory could promote screening and treatment to minimize PTSD symptomatology.

\section{Acknowledgments}

The Acknowledgment is included in the full-text version of this article, available online at www.jco.org. It is not included in the PDF version (via Adobe® Reader®).

Supported by Grant No. R03-CA-101492 from the National Cancer Institute, Doctoral Training Grant in Oncology Social Work Grant No. DSW-0321301-SW from the American Cancer Society, and the University Research Council, University of North Carolina at Chapel Hill.

\section{REFERENCES}

1. American Cancer Society. What are the key statistics about non-Hodgkin's lymphoma?. http://www.cancer.org/docroot/CRI/content/CRI_2_4_1X_What_are_the_key_statistics_for_nonHodgkins_lymphoma_32.asp?sitearea=

2. Cordova MJ, Andrykowski MA, Kenady DE, et al. Frequency and correlates of posttraumatic stress disorder-like symptoms after treatment for breast cancer. J Consult Clin Psychol 1995;63:981-986. [PubMed: 8543720]

3. Kornblith AB, Herndon JE, Weiss RB, et al. Long-term adjustment of survivors of early-stage breast carcinoma, 20 years after adjuvant chemotherapy. Cancer 2003;98:679-689. [PubMed: 12910510]

4. Andrykowski MA, Cordova MJ. Factors associated with PTSD symptoms following treatment for breast cancer: Test of the Andersen model. J Trauma Stress 1998;11:189-203. [PubMed: 9565911]

5. Pittman RK, Lanes DM, Williston SK, et al. Psychophysiologic assessment of posttraumatic stress disorder in breast cancer patients. Psychosomatics 2001;42:133-140. [PubMed: 11239126]

6. Lazarus, RS.; Folkman, S. Stress, Appraisal and Coping. Springer; New York, NY: 1984.

7. Kornblith AB, Powell M, Regan MM, et al. Long-term psychosocial adjustment of older vs. younger survivors of breast and endometrial cancer. Psychooncology 2007;16:895-903. [PubMed: 17245695]

8. Rourke MT, Hobbie WL, Schwartz L, et al. Posttrauamatic stress disorder (PTSD) in young adult survivors of childhood cancer. Pediatr Blood Cancer 2007;49:177-182. [PubMed: 16862538]

9. Dillman, DA. Mail and Internet Surveys: The Tailored Design Method. John Wiley \& Sons; New York, NY: 2000.

10. National Cancer Institute. Adult non-Hodgkin's lymphoma (PDQ): Treatment. http://www.cancer.gov/cancertopics/pdq/treatment/adult-non-hodgkins/HealthProfessional/ page2\#Section_17 
11. Sangha O, Stucki G, Liang MH, et al. The self-administered comorbidity questionnaire: A new method to assess comorbidity for clinical and health services research. Arthritis Rheum 2003;49:156-163. [PubMed: 12687505]

12. University of Minnesota Cancer Center. Childhood cancer survivor study. http://www.cancer.umn.edu/research/programs/peccss.html

13. Sherbourne CD, Stewart AL. The MOS social support survey. Soc Sci Med 1991;32:705-714. [PubMed: 2035047]

14. Ganz PA, Desmond KA, Leedham B, et al. Quality of life in long-term, disease-free survivors of breast cancer: A follow-up study. J Natl Cancer Inst 2002;94:39-49. [PubMed: 11773281]

15. Stuber ML, Christakis DA, Houskamp B, et al. Posttrauma symptoms in childhood leukemia survivors and their parents. Psychosomatics 1996;37:254-261. [PubMed: 8849502]

16. Kornblith AB, Anderson J, Cella DF, et al. Hodgkin's disease survivors at increased risk for problems in psychosocial adaptation. Cancer 1992;70:2214-2224. [PubMed: 1394054]

17. American Psychiatric Association. Diagnostic and Statistical Manual. ed 4. American Psychiatric Association; Washington, DC: 1994.

18. Weathers, FW.; Litz, B.; Herman, D., et al. The PTSD Checklist (PCL-C): Reliability, validity and diagnostic utility. Presented at the 9th Annual Meeting of the International Society for Traumatic Stress Studies; San Antonio, TX. October 24-27, 1993;

19. Blanchard EB, Jones-Alexander J, Buckley TC, et al. Psychometric properties of the PTSD Checklist (PCL). Behav Res Ther 1996;34:669-673. [PubMed: 8870294]

20. Allison, PD. Missing Data. Sage Publications; Thousand Oaks, CA: 2001.

21. SAS Institute Inc. Documentation: The MI-ANALYZE procedure. 2007. update. http://support.sas.com/onlinedoc/913/docMainpage.jsp

22. National Center for Posttraumatic Stress Disorder. How common is PTSD?. http://www.ncptsd.va.gov/ncmain/ncdocs/fact_shts/fs_how_common_is_ptsd.html

23. US Census Bureau. Annual estimates of the population by sex and five-year age groups for the UnitedStates. http://www.census.gov/popest/national/asrh/NC-EST2005/NC-EST2005-01.xls

24. Deimling GT, Kahana B, Bowman KF, et al. Cancer survivorship and psychological distress in later life. Psychooncology 2002;11:479-494. [PubMed: 12476430]

25. Foa EB, Meadows EA. Psychosocial treatments for posttraumatic stress disorder: A critical review. Annu Rev Psychol 1997;48:449-480. [PubMed: 9046566]

26. Kazak AE, Alderfer MA, Streisand R, et al. Treatment of posttraumatic stress symptoms in adolescent survivors of childhood cancer and their families: A randomized clinical trial. J Fam Psychol 2004;18:493-504. [PubMed: 15382974] 


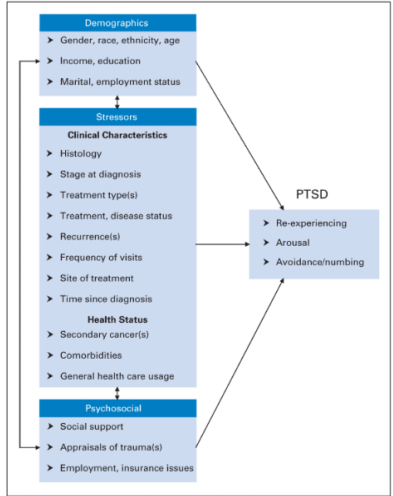

Fig 1.

Conceptual model of cancer-related post-traumatic stress disorder (PTSD). 
Table 1

Characteristics of the Study Sample

\begin{tabular}{|c|c|c|}
\hline Characteristic & $\begin{array}{r}\text { No. of Patients } \\
(\mathbf{N}=\mathbf{8 8 6})\end{array}$ & $\%$ \\
\hline \multicolumn{3}{|l|}{ Demographics } \\
\hline \multicolumn{3}{|l|}{ Sex } \\
\hline Male & 435 & 49.1 \\
\hline Female & 451 & 50.9 \\
\hline \multicolumn{3}{|l|}{ Race } \\
\hline White & 758 & 85.5 \\
\hline African American & 91 & 10.3 \\
\hline Multiple race & 30 & 3.4 \\
\hline Other & 7 & 0.8 \\
\hline \multicolumn{3}{|l|}{ Ethnicity } \\
\hline Non-Hispanic & 873 & 98.5 \\
\hline Hispanic & 13 & 1.5 \\
\hline \multicolumn{3}{|l|}{ Income level } \\
\hline$<\$ 30,000$ & 225 & 25.4 \\
\hline$\$ 30,000-\$ 59,999$ & 239 & 27.0 \\
\hline$\$ 60,000-\$ 89,999$ & 139 & 15.7 \\
\hline$\geq \$ 90,000$ & 189 & 21.3 \\
\hline Unknown & 94 & 10.6 \\
\hline \multicolumn{3}{|l|}{ Education } \\
\hline High school or less & 250 & 28.2 \\
\hline Some college or trade school & 277 & 31.3 \\
\hline College degree & 199 & 22.5 \\
\hline Postgraduate & 138 & 15.6 \\
\hline Unknown & 22 & 2.4 \\
\hline \multicolumn{3}{|l|}{ Marital status } \\
\hline Married & 648 & 73.1 \\
\hline Living with partner & 24 & 2.7 \\
\hline Widowed & 90 & 10.2 \\
\hline Separated/divorced & 48 & 5.4 \\
\hline Single & 67 & 7.6 \\
\hline Unknown & 9 & 1.0 \\
\hline \multicolumn{3}{|l|}{ Employment status } \\
\hline Retired & 477 & 53.8 \\
\hline Employed & 350 & 39.5 \\
\hline Unemployed & 46 & 5.2 \\
\hline Unknown & 13 & 1.5 \\
\hline \multicolumn{3}{|l|}{ Age at enrollment, years } \\
\hline Mean & & 62.9 \\
\hline SD & & 13.5 \\
\hline
\end{tabular}




\begin{tabular}{lrc}
\hline Characteristic & $\begin{array}{r}\text { No. of Patients } \\
(\mathbf{N}=\mathbf{8 8 6})\end{array}$ & $\%$ \\
\hline Range & \multicolumn{2}{c}{$25-92$} \\
$25-49$ & 323 & 17.7 \\
$50-64$ & 315 & 36.5 \\
$65-79$ & 91 & 35.6 \\
$\geq 80$ & & 10.2 \\
Histology of NHL & 445 & \\
Indolent & 391 & 50.2 \\
Aggressive & 50 & 44.2 \\
Unknown & & 5.6 \\
Stage at diagnosis of NHL & & \\
Stage I & 247 & 27.9 \\
Stage II & 159 & 17.9 \\
Stage III & 146 & 16.5 \\
Stage IV & 214 & 24.2 \\
Unknown & 120 & 13.5
\end{tabular}

Types of treatment

Mean, No.

2.1

SD, No.

1.1

No treatment

Surgery

$248 \quad 28.0$

Radiation therapy

$418 \quad 47.1$

Chemotherapy

$692 \quad 78.1$

Bone marrow/stem-cell transplantation

Other therapy

$104 \quad 11.7$

Current treatment status

Not in treatment

Receiving treatment

$117 \quad 13.2$

Unknown

Disease status

In remission or cured
Not in remission
Unknown

$\begin{array}{rl}680 & 76.7 \\ 109 & 12.3 \\ 97 & 11.0\end{array}$

No. of recurrences

0

$\geq 1$

Unknown

No. of NHL-related visits

0

1-2

3-4 


\begin{tabular}{lrr}
\hline Characteristic & $\begin{array}{r}\text { No. of Patients } \\
(\mathbf{N}=\mathbf{8 8 6})\end{array}$ & $\%$ \\
\hline $5-6$ & 131 & 14.8 \\
$7-10$ & 90 & 10.2 \\
$11-20$ & 86 & 9.7 \\
$>20$ & 113 & 12.8 \\
Unknown & 30 & 3.4
\end{tabular}

Site of treatment

Duke University

Age at diagnosis, years

Mean

SD

Range

19-49

50-64

65-79

$\geq 80$

Years since diagnosis

Mean

SD

Range

2-4

5-9

10-14

15-19

$\geq 20$

Clinical characteristics

Secondary cancer(s)

Yes

No

Unknown

Comorbidities, No.

Mean

SD

0

1-2

3-5

6-8

$\geq 9$

Unknown

Years since last physical examination

$<1$ year

$750 \quad 84.7$

$136 \quad 15.3$

52.6

14.2

19-87

$370 \quad 41.8$

$337 \quad 38.0$

$168 \quad 19.0$

$11 \quad 1.2$

10.2

7.1

2-44

$219 \quad 24.7$

$335 \quad 37.8$

$150 \quad 16.9$

88
9

$94 \quad 10.6$

$\begin{array}{rr}120 & 13.5 \\ 752 & 84.9 \\ 14 & 1.6\end{array}$

2.9

2.1

$97 \quad 10.9$

$326 \quad 36.8$

$341 \quad 38.5$

$95 \quad 10.7$

$12 \quad 1.4$

$15 \quad 1.7$

$480 \quad 54.2$ 


\begin{tabular}{|c|c|c|c|}
\hline Characteristic & $\begin{array}{r}\text { No. of Patients } \\
(\mathbf{N}=886)\end{array}$ & & $\%$ \\
\hline 1-2 years & 186 & & 21.0 \\
\hline 3-4 years & 71 & & 8.0 \\
\hline$\geq 5$ years & 128 & & 14.4 \\
\hline Unknown & 21 & & 2.4 \\
\hline \multicolumn{4}{|l|}{ Psychosocial } \\
\hline \multicolumn{4}{|l|}{ Social support } \\
\hline Mean & & 83.1 & \\
\hline SD & & 16.4 & \\
\hline Range & & $20-100$ & \\
\hline \multicolumn{4}{|c|}{$\begin{array}{l}\text { Appraisal of life threat and treatment } \\
\text { intensity }\end{array}$} \\
\hline Mean & & 19.3 & \\
\hline SD & & 6.0 & \\
\hline Range & & $6-30$ & \\
\hline \multicolumn{4}{|c|}{$\begin{array}{l}\text { Employment and insurance issues } \\
\text { related to cancer }\end{array}$} \\
\hline Mean & & 1.1 & \\
\hline SD & & 2.1 & \\
\hline Range & & $0-17$ & \\
\hline
\end{tabular}

Abbreviations: SD, standard deviation; NHL, non-Hodgkin's lymphoma; UNC, University of North Carolina. 
Table 2

Prevalence of Cancer-Related PTSD $(\mathrm{N}=868)$

\begin{tabular}{|c|c|c|c|c|}
\hline \multirow[b]{2}{*}{ Indicator of PTSD* } & \multicolumn{2}{|r|}{ Nonadjusted } & \multicolumn{2}{|c|}{$\begin{array}{r}\text { Adjusted for } \\
\text { Nonresponse }^{\dagger}\end{array}$} \\
\hline & $\%$ & $95 \% \mathrm{CI}$ & $\%$ & $95 \% \mathrm{CI}$ \\
\hline Score $\geq 44$ & 6.9 & 5.2 to 8.6 & 7.4 & 5.6 to 9.1 \\
\hline 3 Symptom clusters & 7.6 & 5.8 to 9.4 & 7.9 & 6.1 to 9.7 \\
\hline 2 Symptom clusters & 8.9 & 7.0 to 10.8 & 9.1 & 7.2 to 11.0 \\
\hline 1 Symptom cluster & 22.2 & 19.5 to 25.0 & 22.3 & 19.5 to 25.0 \\
\hline No symptom clusters & 61.3 & 58.1 to 64.5 & 60.7 & 57.5 to 64.0 \\
\hline
\end{tabular}

Abbreviation: PTSD, post-traumatic stress disorder.

* Based on the PTSD Checklist-Civilian Version (PCL-C). Symptom clusters include $\geq$ three avoidance symptoms, $\geq$ two arousal symptoms, and $\geq$ one re-experiencing symptom. All three clusters constitute full PTSD.

${ }^{\dagger}$ Adjusted for current age, age at diagnosis, and race. 


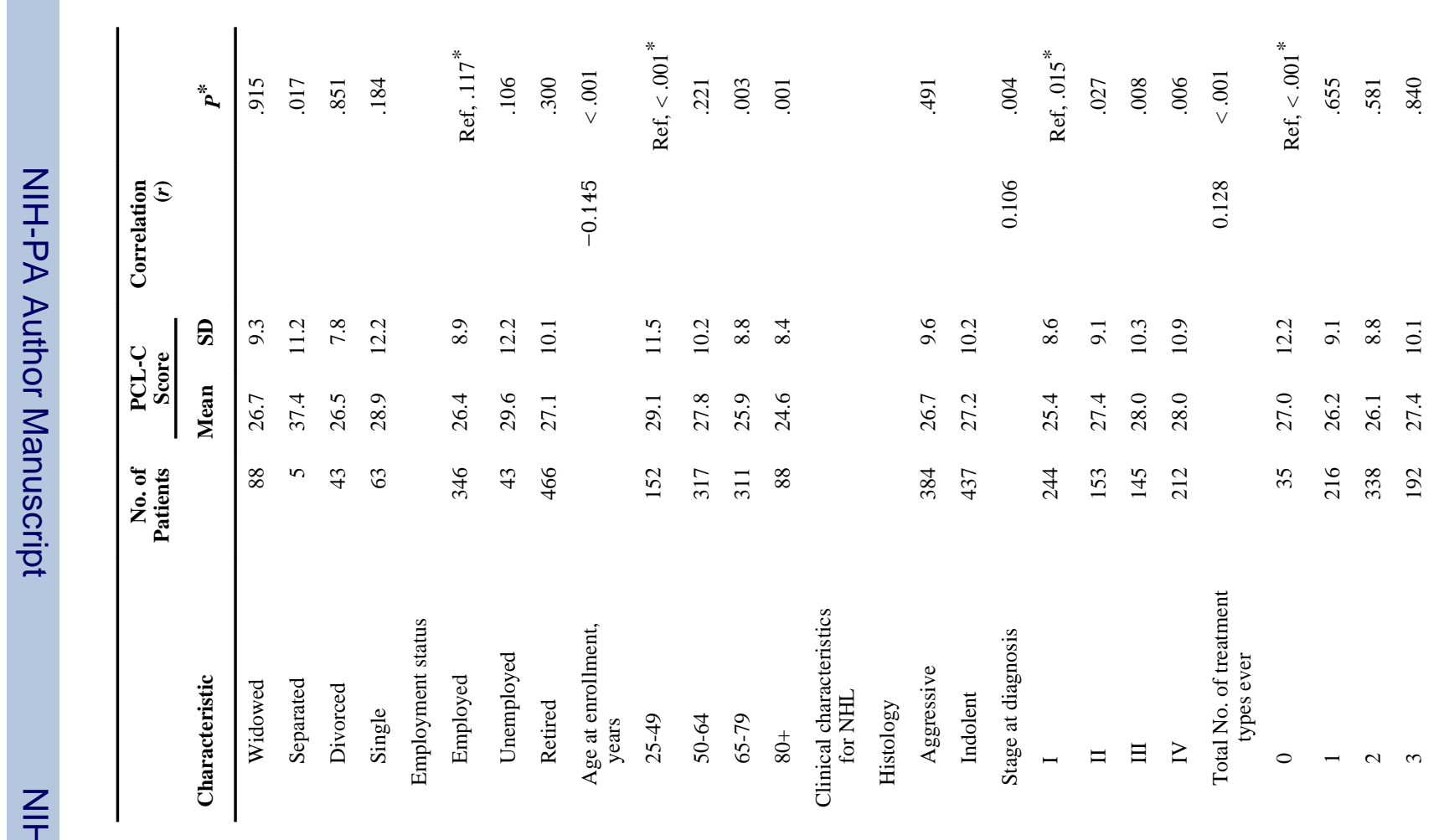




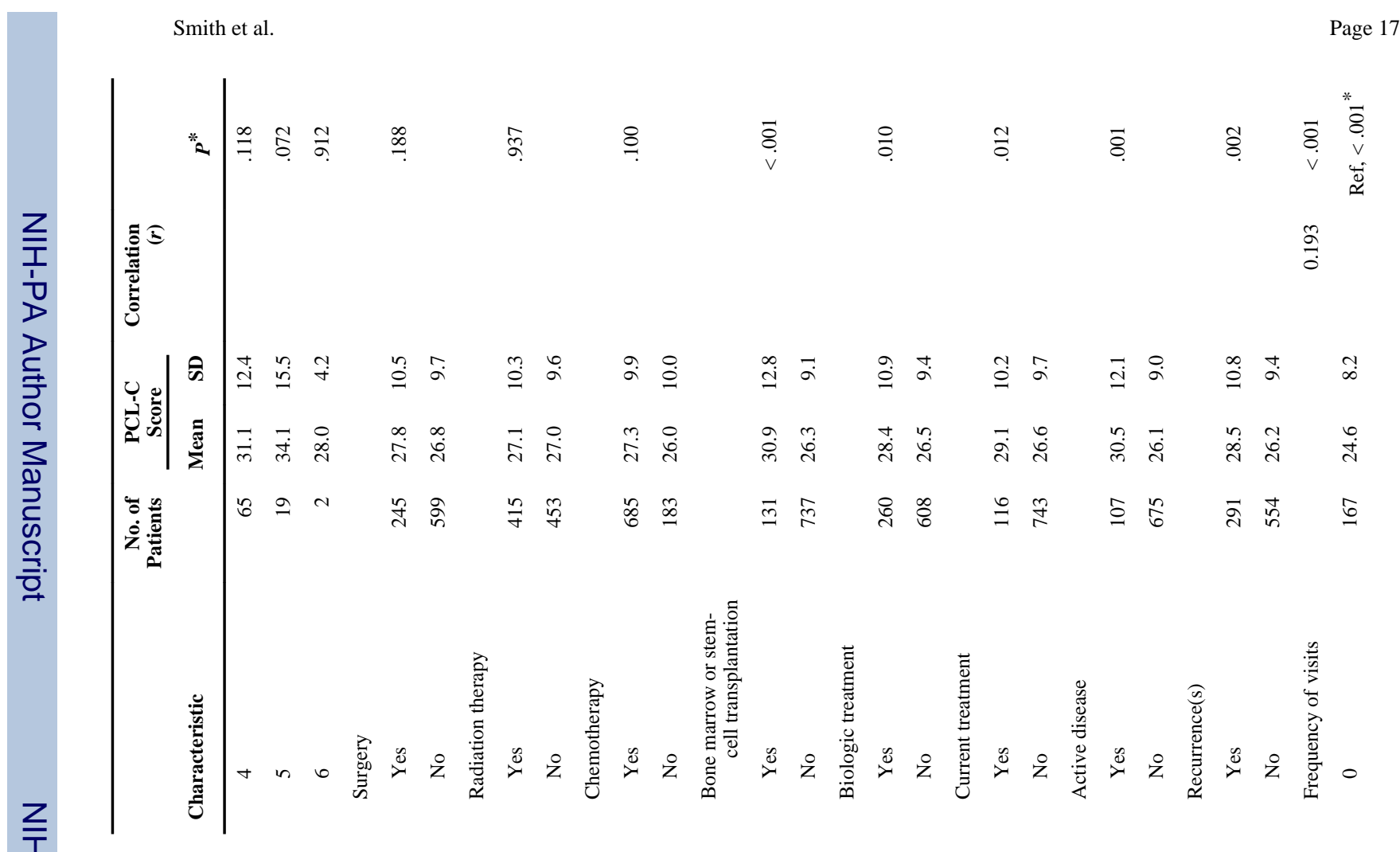




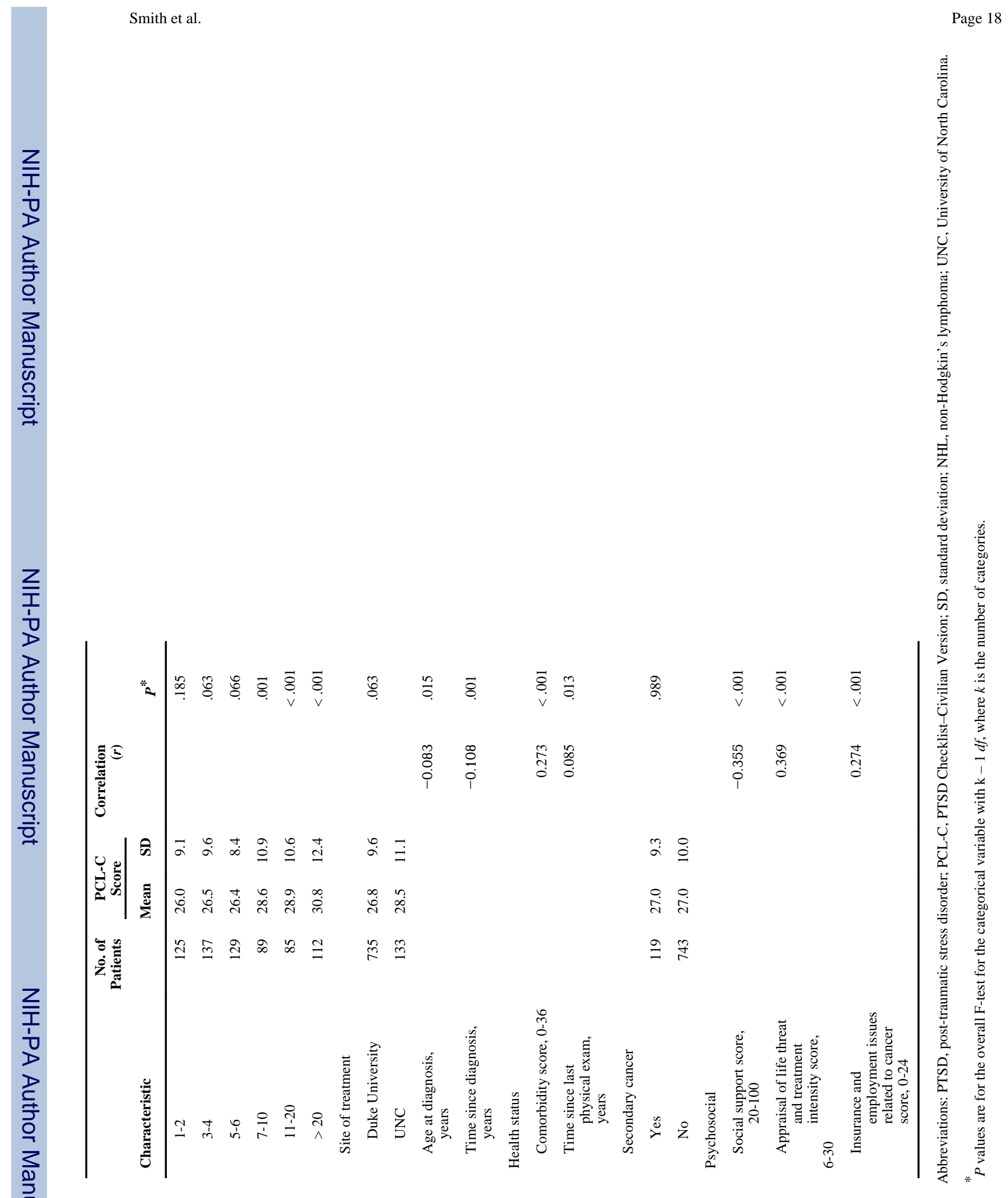

J Clin Oncol. Author manuscript; available in PMC 2011 January 24. 


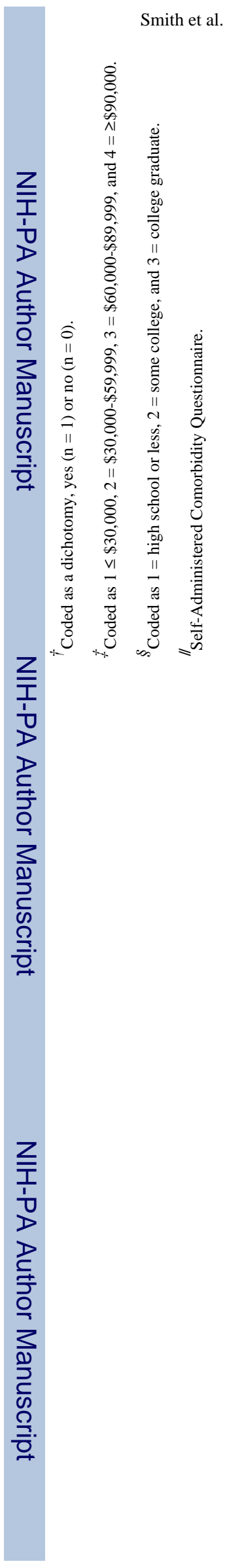

